\title{
Radiolabeled Nanoparticles in Nuclear Oncology
}

\author{
E.A. Salvanou, P. Bouziotis, C. Tsoukalas* \\ Radiochemical Studies Laboratory, Institute of Nuclear and Radiological Sciences \& Technology, Energy \& \\ Safety, National Center for Scientific Research "Demokritos”, Athens, Greece \\ *Corresponding author email: ctsoukal@rrp.demokritos.gr
}

Received: 2 April 2018 / Accepted: 9 April 2018 / Published: 22 April 2018

\begin{abstract}
During recent years, a plethora of pioneering radiolabeled nanoparticles have grown to be an integral part of nuclear medicine as theranostic tools. Herein, we focus on the most representative examples of nanoparticles of the past decade, which have been investigated in conjunction with radioisotopes aiming to serve as drug delivery or imaging agents. The present review highlights the key attributes of each nanosystem and the following classification of radiolabeled nanovehicles is based on increasing mass number (A) of radioisotopic elements.
\end{abstract}

Keywords: Cancer, diagnosis, MRI, nanooncology, nanoparticle, nuclear imaging, PET, radioisotope, SPECT, theranostics, therapy

\section{Introduction}

Cancer is a generic term for a large group of diseases characterized by the uncontrolled growth and spread of abnormal cells, caused by an interaction between external factors and mutations on a molecular level, both of which are yet to be fully understood. Certain types of cancer along with stroke, ischaemic heart and respiratory diseases are classified by the World Health Organization (WHO) in the top 5 of leading causes of morbidity and mortality worldwide for the year 2015 [1]. According to the last report of "Globocan Project" of the International Agency for Research on Cancer (IARC) of WHO, 14.1 million new cancer cases, 32.6 million people living with cancer and 8.2 million deaths worldwide were estimated for the year 2012 [2]. The number of new cases is estimated to reach the staggering number of 23.97 million over the next 2 decades. According to deaths recorded in 2015 and by simple calculations, approximately 17 people per minute succumb to this devastating disease worldwide.

In the last decades, a considerable number of scientific disciplines and especially nanomedicine have flourished due to the emergence of advanced novel nanomaterials. Therapeutic and diagnostic tools based on nanotechnology have come to bring the revolution in current medical practice and the solution to problems that remain still unsolved. Due to the need of dealing with medical issues, such as cancer, nanoparticles have gained tremendous interest. The main role of a nanocarrier is targeted delivery of a drug directly into the ailing area, preventing the undesired side-effects of conventional medicines and their uncontrolled biodistribution. Since tumors and metastases are highly heterogenous, efficacy of nanomedicines has significant intra-individual difference. Various surface functionalization methods have been devised to influence advantageous features of nanoplatforms. In some cases, nanoparticles offer the possibility of additional active targeting methods by conjugating a peptide or an antibody fragment, as well as improved therapeutic effect by combining several different methods: chemotherapy; radioisotope therapy (RIT); magnetic hyperthermia (MHT); photodynamic therapy (PDT); photothermal therapy (PTT). 
Radiolabeled Nanoparticles in Nuclear Oncology

Alternative methods of treatment include surgery, radiation, chemotherapy, hormone therapy, immune therapy, and targeted therapy.

Radioisotopes have grown to be an integral part of the field of medical applications both for purposes of drug development and now diagnostic and therapeutic applications. Multivalency provides NPs with the required flexibility to enable the utilization of radioisotopes in novel applications. Careful construction and evaluation of these NP probes using nuclear imaging methods provides considerable insight into their in vivo fate and their prospective utility in research and clinical application. Convergence of radiolabeling with the cornucopia of NPs for imaging and therapy has resulted in many particles capable of multimodal imaging. For cancer diagnosis, the list of imaging modalities includes Magnetic Resonance Imaging (MRI), Computed Tomography (CT), Positron Emission Tomography (PET), Single Photon Emission Computed Tomography (SPECT), Optical Imaging (OI) and Cerenkov Luminescence (CL). A very useful imaging tool for clinical oncology is also the fused form of nuclear and anatomical images from CT and SPECT (SPECT/CT) or PET (PET/CT) into a single device. Molecular imaging of cancer is crucial, therefore almost every permutation of the various imaging modalities in combination with nanoparticle carriers is actively investigated. Recent advances in tumor imaging technology has opened the possibility of early detection of the disease, resulting in a more successful way of treatment. The most recent literature is mentioned, providing a classification of radiolabeled nanovehicles based on increasing mass number (A) of radioisotopic elements.

\section{Radiolabeled Materials}

\subsection{Carbon-11}

A strategy for labeling different nanoplatforms via covalent bonding with ${ }^{11} \mathrm{C}$, in order to develop a useful tool for PET and MR imaging, was reported by Sharma et al (2013). In their study, they examined nanoparticles appropriately functionalized with amine $\left(-\mathrm{NH}_{2}\right)$ and carboxylic acid $(-\mathrm{COOH})$ groups and more precisely iron oxide nanoparticles (IONPs), and platinum and silicon dioxide nanoparticles. It was proved that all functionalized nanoconstructs could bond efficiently with the radioisotope and provide sufficient radioactivity to perform PET imaging and biodistribution studies [3].

\section{$2.2 \quad$ Fluorine-18}

Inorganic materials like hydroxyapatite $(\mathrm{HA})$ and aluminium hydroxide $\left(\mathrm{Al}(\mathrm{OH})_{3}\right)$ have been used to efficiently provide labeled radiotracers with $\left[{ }^{18} \mathrm{~F}\right]$-fluoride for in vivo imaging. These radiotracers, which demonstrated different in vivo behavior depending on the route of administration, were monitored by PET imaging and showed uptake in the joints of the skeleton. Once further modified, these nanoparticles could lead to targeted contrast agents with lower in vivo aggregation and uptake in the RES organs [4].

Gold nanoparticles, functionalized with peptides and bonded covalently to $\mathrm{N}$-succinimidyl-4-[18 $\mathrm{F}]$ fluorobenzoate $\left(\left[{ }^{18} \mathrm{~F}\right]-\mathrm{SFB}\right)$, have been synthesized. Their in vivo biodistribution was assessed and high accumulation in the bladder and urine, and low intestinal uptake were demonstrated. Ex vivo biodistribution revealed accumulation in the RES organs which could be due to the negative surface charge of the nanoparticles, resulting in phagocytisis by macrophages of these organs [5]. A fast and simple strategy of radiolabeling and coating magnetic nanoparticles was introduced by Sun Z. et al (2016). A polymer coating made of polyacrylic acid (PAA) and oleylamines and the chelator S-2-(4-aminobenzyl)-1,4,7triazacyclononane-1,4,7-triacetic acid ( $\mathrm{p}-\mathrm{NH}_{2}$-Bn-NOTA) were applied, rendering these IONPs efficient PET/MR imaging probes [6]. ${ }^{18} \mathrm{~F}$-labeled dipalmitoyl $\left[{ }^{18} \mathrm{~F}\right]-\mathrm{FDP}$ liposomes were reacted with tumor tissues, demonstrating increased uptake. The reaction between tetrazine and trans-cyclooctenes was exploited in order to attain the rapid delivery of biorthogonal tetrazine radiolabeled lisposomes to tumors, in relation to liposomes without the tetrazine label [7].

Labeling strategies of polymeric nanoparticles with $4-\left[{ }^{18} \mathrm{~F}\right]$ fluorobenzyl-2-bromoacetamide $\left(\left[{ }^{18} \mathrm{~F}\right] \mathrm{FBBA}\right)$ were investigated and based on the rationale of radiolabeling a precursor block-copolymer and then incorporating it as constituent element onto other polymeric nanoparticles. Three different approaches 
were tried; two of them consisted of two different radiolabeled block-copolymers, while the third one utilized $\left.{ }^{18} \mathrm{~F}\right] \mathrm{FBBA}$ entrapped in the complex. All strategies included a targeting AGBBB015F (15F) peptide, grafted onto the NP surface. Biodistribution profiles and PET/CT imaging were evaluated in tumorbearing rodents, and exhibited low accumulation in the liver and renal excretion in principal [8]. A very recent study investigated the possibilities offered by an organosilicon fluoride acceptor (SiFA) in conjunction with the synthesis of polymeric core-shell nanoparticles. In this study, nanoparticles of four distinct sizes were synthesized, functionalized and radiolabeled using a very facile and easily repeated method, without additional chemical modification. The nanosystems with a hydrodynamic diameter of 33nm exhibited the best results in EMT6 tumor-bearing mice [9].

\section{$2.3 \quad$ Copper-64}

Liposomes of various sizes were synthesized, doped with 1,2-distearoyl-sn-glycero-3-phosphocholine (DSPC) which was functionalized with the 1,4,7,10-tetraazacyclododecane-1,4,7,10-tetraacetic acid (DOTA) chelator for ${ }^{64} \mathrm{Cu}$ labeling and evaluated as potential PET radiotracer for bone marrow imaging. Higher accumulation in the bone marrow was revealed for the smaller-sized liposomes in comparison to larger-sized ones, at $24 \mathrm{~h}$ post-injection. Tumor accumulation in animal models was unequivocally smaller than accumulation in the bone marrow [10]. Furthermore, Lee H. et al (2015) developed another method for ${ }^{64} \mathrm{Cu}$ radiolabeling via the gradient loadable chelator 4-DEAP-ATSC encapsulated into PLD (liposomal doxorubicin (DOX) decorated with polyethylene glycol chains) and PLD targeted with HER2. In vitro as well as in vivo stability studies exhibited high stability up to $48 \mathrm{~h}(>99 \%)$ and $24 \mathrm{~h}(>94 \%)$ respectively. PET/CT imaging in BT474-M3 xenografts substantiated the use of these radiolabeled nanoparticles as PET tracers for in vivo liposome tracking [11]. A more recent study utilized DOX-loaded liposomes capable of PET and NIFR imaging after conjugation with ${ }^{64} \mathrm{Cu}$ and IRDye $800 \mathrm{CW}$ respectively. A PD-1 (programmed cell death-1) monoclonal antibody was also added onto this nanoconstruct, enabling 4T1 tumor visualization and treatment [12]. Lipid nanoparticles (LNPs), containing a distearoyl phosphatidyl ethanolamine monomethoxy polyethylene glycol (DSPE-PEG)-maleimide co-polymer, were assembled and covalently linked to an anti-prostate membrane antigen (PSMA) single chain ( $\mathrm{scFv}$ ) antibody with cysteine, enabling active targeting. Later on, the DOTA chelator was added to offer radiolabeling with ${ }^{64} \mathrm{Cu}$. Through this research, it was proved that $\mathrm{scFv}$ antibodies could be utilized as therapeutic tools due to their potential of conjugation with nanoparticles as they offered enhanced anti-tumor targeting [13].

Pang B. et al (2015) designed PdCu tripods with a conformal Au shell. This well-structured platform was radiolabeled by substitution of some of the $\mathrm{Cu}$ particles of the core with the radioactive counterpart. After conjugation with D-Ala1-peptide T-amide (DAPTA), the tripods could serve as an efficient platform for PET imaging and photothermal treatment for triple negative breast cancer (TNBC) with enhanced anticancer properties, as proved by the in vivo results [14]. In another study, a controllable layer of gold nanoparticles was used to encapsulate ${ }^{64} \mathrm{Cu}$ ions, using a chelator free process. The highly stable construct was studied in vivo with PET imaging in tumor-bearing animal models with three different coatings (1dodecanethiol and Tween 20; MeO-PEG5000-SH; and sodium 10-mercaptodecane-sulfonic acid and (10mercaptodecyl)trimethylammonium bromide)), resulting in PEG-coated $\mathrm{Au}$ nanoparticles which demonstrated longer circulation time and enhanced tissue uptake compared to the other two coatings examined [15]. Radiolabeled Au nanorods have also been used to study in vivo kinetic behavior with regard to their size. These nanorods are delivered to the tumorous site via the enhanced permeability and retention (EPR) effect [16]. Nanoclusters (NCs) have also started to be thoroughly investigated in cancer diagnostics, as was the case when Zhao Y. et al (2017) utilized Au NCs for breast cancer and metastasis recognition. Targeting of the overexpressed CXCR4 receptor and consequent PET imaging was possible after doping with ${ }^{64} \mathrm{Cu}$ and functionalizing the AuNCs with plerixafor. Eventually the pharmacokinetics of these complexes were assessed, and high sensitivity and accuracy in early-stage tumor detection was reported [17]. Glycol chitosan nanoparticles (CNPs) were synthesized with a copper-free click chemistry method aiming to prove that such a method would facilitate a preradiolabeling pathway for the in vivo assessment of 
Radiolabeled Nanoparticles in Nuclear Oncology

radiolabeled nanoparticles. Following this synthetic pathway, a DCBO-PEG 4 -Lys-DOTA- ${ }^{64} \mathrm{Cu}$ complex was formed, where dibenzyl cyclooctyne (DBCO) was conjugated to a DOTA chelator for labeling with ${ }^{64} \mathrm{Cu}$, which was then incubated with azide-functionalized CNPs, resulting in their successful radiolabeling at high radiochemical yields [18].

Silica nanoparticles, functionalized with a soft electron-donating thiol group, were synthesized by Shaffer et al (2016) in order to achieve a thermodynamically stable bond with ${ }^{64} \mathrm{Cu}$ without chelation and expand their application of silica NPs in molecular imaging using both hard and soft radiometal ions [19].

A common strategy for the development of radiolabeled superparamagnetic iron oxide nanoparticles (SPIONs) is the direct labeling of their inorganic surface with an agent that binds to its surface as well as with the isotope without affecting the polymeric coating. In this regard, a bisphosphonate agent was modified to contain dithiocarbamate and served as a chelator for the binding with ${ }^{64} \mathrm{Cu}$ and consequent binding to SPIONs, namely the commercially available Endorem/Feridex. In vitro studies demonstrated sufficient binding for at least 48h, while PET-CT and MRI studies revealed uptake in the popliteal lymph nodes and the iliac lymph nodes, to a lesser extent [20]. In another study, SPIONs utilized as vehicles for an anticancer agent (doxorubicin) via a $\mathrm{pH}$-sensitive bond were decorated with PEG chains, the cyclo(ArgGly-Asp-D-Phe-Cys) peptide (c(RGDfC)) for tumor targeting and 1,4,7-triazacyclononane-N,N',N"triacetic acid (NOTA) chelator for radiolabeling. This approach resulted in a cytotoxic nanocarrier capable of PET and MR imaging by efficiently targeting $\alpha_{\nu} \beta_{3}$-expressing tumors [21].

Radiolabeled graphene oxide (GO) nanoparticles were exploited as imaging agents as well as drug delivery vehicles in breast cancer lung metastasis animal models. Radiolabeling was accomplished via a NOTA chelating agent, while a monoclonal antibody (mAb) was used to drive these carriers to the folliclestimulating hormone receptor (FSHR). DOX was sufficiently loaded to the carriers and successfully delivered to tumorous sites, in contrast with the untargeted NPs [22]. Manganese oxide nanoparticles $\left(\mathrm{Mn}_{3} \mathrm{O}_{4}\right)$ could be useful diagnostic tools for PET and MR imaging. Similarly to the GO NPs mentioned above, the $\mathrm{Mn}_{3} \mathrm{O}_{4}$ nanoparticles were coated with PEG, labeled with ${ }^{64} \mathrm{Cu}$ via the NOTA chelator and actively targeted with the addition of the TRC105 antibody. The enhanced targeting specificity of the nanocomplex towards 4T1 breast cancer was advocated by all the experimental results of this study [23].

\section{$2.4 \quad$ Gallium-68}

AGuIX are ultrasmall (diameter $<5 \mathrm{~nm}$ ) rigid multimodal imaging platforms constituted of a polysiloxane matrix scaffold labeled with both gadolinium $\left(\mathrm{Gd}^{3+}\right)$ and ${ }^{68} \mathrm{Ga}$ via adequate chelators, which could serve as a PET/MR imaging agent. Initial work with DOTA as the chelating agent showed promising results, as residual activity on all untargeted tissues was extremely low. The initial encouraging results led Truillet et al (2015) to functionalize these NPs with chelators such as DOTAGA (1,4,7,10-tetraazacyclododecane-1glutaric anhydride-4,7,10-triacetic acid) [24] and NODAGA (2,2'-(7-(1-carboxy-4-((2,5-dioxopyrrolidin-1yl)oxy)-4-oxobutyl)-1,4,7-triazonane-1,4-diyl)diacetic acid)) [24], [25], for improved radiolabeling. It was demonstrated that a single-injection with ${ }^{68} \mathrm{Ga}-\mathrm{AGuIX} @$ NODAGA nanoparticles could provide a dualmodality imaging agent adequate for both PET and MRI.

Small-sized gold nanoparticles $(2 \mathrm{~nm})$ were coated with glucose and conjugated with the NOTA chelating agent for further radiolabeling with ${ }^{68} \mathrm{Ga}$. The aim of this nanosystem was to explore the blood brain barrier permeability (BBB) with PET imaging. Therefore, targeted and non-targeted systems were prepared in the presence or absence of BBB-permeable neuropeptides. A 3-fold higher brain accumulation of the targeted AuNPs was shown by the in vivo biodistribution studies [26]. Iron Oxide nanorods, coated with silica and various ratios of PEG and DOTA chelator were assessed as potential PET/MR imaging agents. All silica coated iron oxide nanorods formed highly stable radiolabeled complexes, both with and without chelator. Furthermore, it was shown that PEG-coated nanorods were also capable of direct radiolabeling within 15 minutes of incubation with ${ }^{68} \mathrm{Ga}$, with high in vivo stability, suitable for PET/MR imaging of liver malignancies [27]. In the same direction, Moon S.H. et al (2016), encapsulated iron oxide nanoparticles with three amphiphiles containing PEG, the DOTA chelator for ${ }^{68} \mathrm{G}$ a radiolabeling and the prostate-specific 
membrane antigen (PSMA) for tumor targeting. MR imaging studies resulted in uptake by only PSMA positive tumor cells in dual-tumor animal model, which was also confirmed by PET imaging [28]. Another dual-modality imaging probe was devised from the same group for the targeting of lymph nodes, with encapsulation of IONPs in amphiphiles containing NOTA and D-mannose, the specificity of which was confirmed with PET and MR imaging [29].

Graphene oxide (GO) nanosheets were hybridized with ${ }^{68} \mathrm{Ga}$-magnetite $\left(\mathrm{Fe}_{3} \mathrm{O}_{4}\right)$ NPs, forming a radiolabeled magnetic graphene oxide (MGO) nanocomposite that can be applied as a drug delivery system. No detachment of ${ }^{68} \mathrm{G}$ a from the complex was shown in in vitro stability studies, while in vivo biodistribution studies revealed high uptake by vital organs and fast clearance from the kidneys, as well as reduced NP uptake by the RES organs, which can be attributed to GO grafting of magnetite NPs. Consequently, ${ }^{8} \mathrm{Ga}-$ MGO could serve as promising agent for PET imaging [30]. In the aim of assessing porous zirconia NPs as drug delivery nanoplatforms, these were functionalized with the DOTA chelator and consequently radiolabeled with ${ }^{68} \mathrm{Ga}$ at high radiolabeling yields. In vitro studies demonstrated a very stable radiolabeled complex while $\mu$ PET/CT results showed higher uptake of the NPs in the RES organs, low uptake in the lungs indicating the lack of aggregates, extended blood retention times and slow kidney clearance [31].

\subsection{Arsenic-71, 72, 74, 76}

Although As radioisotopes are not widely used because of limited availability and difficulties related to their production, separation and purification of the radioisotopes [32], Chen F. et al (2013) employed a simple, chelator-free and efficient protocol to synthesize *As-radiolabeled SPIONs functionalized with a layer of oleic acid (OA) surfactant which was later replaced by poly(acrylic acid) (PAA), leading to higher stability of the NPs in biological solutions. Further coating with PEG made the system more stable, showing high liver uptake and significantly decreased bladder uptake, results also confirmed by PET imaging [33].

\subsection{Zirconium-89}

In the study of Karmani et al, gold nanoparticles with a mean size of $5 \mathrm{~nm}$ were functionalized with the monoclonal antibody cetuximab, which was first radiolabeled with ${ }^{89} \mathrm{Zr}$ via a desferal moiety. The team used a new method for the synthesis, compared the biodistribution before and after the coupling reaction and evaluated quantitive PET imaging performance of the complexes. In vitro studies showed that all conjugates were stable, while in vivo distribution showed no alteration in tumor uptake between the conjugated and unconjugated cetuximab, indicating that ${ }^{89} \mathrm{Zr}$-labeled cetuximab-targeted nanoparticles preserved their tumor targeting properties [34]. Ultra-small paramagnetic iron oxide nanoparticles (USPIOs) were developed to create stable and chelate-free radiolabeled complexes using a novel reaction process. ${ }^{89} \mathrm{Zr}$ was bound to the surface of the magnetite core, through ionic interactions of the opposite charges between the metal ions and the surface layer, resulting in radiolabeling yields over $90 \%$. PET/CT imaging studies were performed, confirming the ability of these nanoplatforms to be radiolabeled with other radioisotopes (i.e. $\left.{ }^{64} \mathrm{Cu}^{2+},{ }^{89} \mathrm{Zr}^{4+},{ }^{111} \mathrm{In}^{3+}\right)$ under the same radiolabeling conditions [35]. In another study, ferumoxytol was linked to the desferrioxamine chelate (DFO) and radiolabeled with ${ }^{89} \mathrm{Zr}$, in order to acquire a tool that could combine PET and MR imaging for the enhanced detection of lymphatic drainage [36].

Imaging of the lymphatic drainage with PET and MRI was also the objective of a recent study, where metal oxide $\left(\mathrm{M}_{\mathrm{x}} \mathrm{O}_{\mathrm{y}}\right)$ nanoplatforms were coated with PEG and radiolabeled with a chelator-free process. They developed constructs with 10 different types of $\mathrm{M}_{\mathrm{x}} \mathrm{O}_{\mathrm{y}}$ and selected the gadolinium (Gd) containing nanoplatform for further testing [37]. Nanoscale metal-organic frameworks (nMOF) materials were investigated as drug delivery vehicles capable of fluorescent and PET imaging for negative breast neoplasia. These octahedron-shaped nanocarriers were labeled with ${ }^{89} \mathrm{Zr}$, decorated with pyrene-derived polyethylene glycol (Py-PGA-PEG) chains, actively targeted with F3 ligand and loaded with DOX. The results were encouraging as there was no toxicity referred to the experimental murine models [38].

A chelator-free system of radiolabeled mesoporous silica nanoparticles (MSN) was presented attaining a long-term in vivo stability of more than 20 days. Both radiolabeling and in vivo stability appeared to be 
Radiolabeled Nanoparticles in Nuclear Oncology

interwoven with the presence of deprotonated silanol groups (-Si-O-) inside or on the surface of the nanoparticles. The conclusion of this study was that ${ }^{89} \mathrm{Zr}-\mathrm{MSN}$ could be a useful tool for tracking the in vivo fate and PET imaging of drug delivery systems [39]. Yet another ${ }^{89} \mathrm{Zr}$ PET tracer was developed by radiolabeling ultrasmall fluorescent silica nanoparticles (C dots) using two pathways: a chelator-free radiolabeling method and a DFO chelating agent. Both nanoplatforms had an integrin-targeting peptide (cRGDY) attached and were coated with PEG. Their stability, pharmacokinetics and uptake were investigated in human melanoma models with encouraging results [36].

Liposomal nanoparticles were developed and efficiently radiolabeled with ${ }^{89} \mathrm{Zr}$ using a ligand exchange reaction. Three versatile vehicles encapsulating desferrioxamine (DFO) were produced, all with high radiochemical yields, one of which was PEGylated while the other one was actively targeted with the aid of folic acid (FA). Comparative studies pertaining to their stability, cell uptake and in vivo pharmacokinetics were performed [40].

Truillet et al (2016) described the synthesis of AGuIX nanoparticles functionalized with DFO, which were radiolabeled with ${ }^{89} \mathrm{Zr}$ at high yields ( $~ 99 \%$ yield), in order to evaluate the magnitude and pharmacokinetics of the long-lived radioisotope within the tumor microenvironment through in vitro, in vivo and PET imaging studies [41]. The group of Groult H. et al (2015) performed very extensive research pertaining to the facile functionalization of 3 different types of nanoparticles, using a modular approach and exploiting the high versatile and binding capabilities of bovine serum albumin (BSA). IONPs, upconverting nanophosphors and $\mathrm{Au}$ nanospheres were used in this study in conjunction with various chelating agents appropriate for the conjugation either with ${ }^{68} \mathrm{Ga}$ or ${ }^{89} \mathrm{Zr}$. Finally, the IONPs functionalized with BSA and modified with DFO were the chosen platform for further investigation. This nanoconstruct was then conjugated to RGD peptide and labeled with ${ }^{89} \mathrm{Zr}$ to offer PET/CT and MR imaging capabilities [42].

\subsection{Yttrium-90}

For therapeutic purposes, ${ }^{90} \mathrm{Y}$ was used to radiolabel magnetic $\left(\mathrm{Fe}_{3} \mathrm{O}_{4}\right)$ nanoparticles with or without a poly(ethylene glycol) 600 diacid coating. The PEG-coated nanoparticles exhibited excellent in vitro stability and lower uptake in the lungs compared to the naked magnetic nanoparticles. The latter could be attributed to the presence of poly(ethylene glycol), which is an inhibitory factor of agglomeration of MNPs in vivo [43]. Magnetic microspheres, coated with citric acid and encapsulated with human serum albumin were produced for application in bimodal radioisotope and hyperthermia cancer treatment. These nanoplatforms were radiolabeled with ${ }^{90} \mathrm{Y}$ using a chelator-free protocol, and provided high in vitro stability and sufficient heating efficiency in order to induce cell apoptosis [44].

\subsection{Technetium-99m}

Curcumin-loaded solid lipid nanoparticles were used to circumvent the low solubility and consequently the low bioavailability of curcumin, which is of particular value due to its anti-oxidant and anti-carcinogenic properties. Liver-spleen imaging with this nanosystem was found to be advantageous and potentially therapeutic [45]. Lipid nanocarriers were also developed by Ucar E. et al (2016), who loaded them with paclitaxel (PTX) and radiolabeled them using the ${ }^{99 \mathrm{~m} T c}(\mathrm{CO}) 3_{3}{ }^{+}$moiety. Active targeting of folate-expressing tumors was efficiently achieved by surface modification with a folate derivative, as proved by the in vivo animal studies [46].

Almost a decade ago, poly(lactic-co-glycolic acid) (PLGA-based nanoparticles labeled with ${ }^{99 \mathrm{~m}}$ Tc in a direct approach were developed, in order to cross the Blood Brain Barrier (BBB). These constructs showed promising results when loaded with chloramphenicol (CHL) and decorated with polysorbate-80 (PS-80) surfactant, as uptake in the brain was higher than that in the bone marrow [47]. The group of Subramanian et al (2010) directly radiolabeled PLGA nanoparticles, utilizing $\mathrm{SnCl}_{2}$ as the reducing agent, and investigated their application in sentinel lymph node detection (SLND), showing localization in the SLN, albeit lower than the uptake observed for the commercially available for SLN detection radiopharmaceutical Nanocis [48]. A few years later, the same group utilized the PLGA nanoparticles mentioned above in two different 
ways, one by encapsulating mebrofenin in situ and another by conjugating p-aminobenzyl diethylenetriamine pentaacetic acid ( $\left.\mathrm{p}-\mathrm{NH}_{2}-\mathrm{Bz}-\mathrm{DTPA}\right)$. The latter functionalization led to improved radiolabeling yields under milder reaction conditions and improved uptake in the SLN [49]. A common palliative treatment for bone metastasis with satisfactory results is the use of ethylenediamine-tetramethylenephosphonic acid (EDTMP) labeled with the radioisotope ${ }^{153} \mathrm{Sm}$. The conjugation of this acid with polymeric nanoparticles consisting of polylactic acid (PLA) and polyvinyl alcohol (PVA) was designed in an attempt to overcome the drawbacks of ${ }^{153} \mathrm{Sm}$-EDTMP. Indeed, biodistribution analyses conducted on these nanoparticles, after their radiolabeling with ${ }^{99 \mathrm{~m}} \mathrm{Tc}$, came to prove this theory, as increased accumulation of these ${ }^{99 \mathrm{~m} T \mathrm{~T}-}$ PLA/PVA/EDTMP complexes in osseous tissues was observed [50].

Gold nanoparticles were also developed as potential SLN imaging agents which remained within the first lymph node for $24 \mathrm{~h}$, with negligible uptake in all other tissues and minimal accumulation in the kidney [51]. This was pursued by conjugating $\mathrm{Au}$ nanoparticles to the hydrazinonicotinamide-Gly-Gly-Cys- $\mathrm{NH}_{2}$ (HYNIC-GGC) peptide, a thiol-triazole-mannose derivative and EDDA/tricine coligand. Furthermore, in order to achieve GRP-r imaging, gold NPs were functionalized with Lys ${ }^{3}$-bombesin and radiolabeled with ${ }^{99 \mathrm{~m} T \mathrm{Tc} \text {. Biodistribution studies showed maximum tumor uptake of the radiotracer at } 1 \mathrm{~h} \text { post-injection [52] }}$ The above results encouraged Ocampo-Garcia et al (2011) to formulate a kit for facile labeling of AuNPs conjugated to Lys ${ }^{3}$-bombesin, cyclo[Arg-Gly-Asp-D-Phe-Lys-(Cys)] or thiol-mannose, which is important as the development of such kits could be a useful tool for the preparation of radiolabeled NPs used for target-specific applications in the clinic [53]. The most recent study on Au nanoparticles radiolabeled with ${ }^{99 \mathrm{~m} T c}$ radioisotope was conducted by Zhao L. et al (2018), who entrapped the nanoparticles in polyethylenimine (PEI) so as to obtain a dual-modality imaging probe. PEI offered versatility for further conjugation with the DTPA chelator and a PEG monomethyl ether, aiming to improve the properties of the nanoconstruct, namely stability, biocompatibility and imaging performance in SPECT/CT imaging of lymph nodes and other organs [54].

Ultra-small paramagnetic iron oxide nanoparticles were prepared along with a PEG polymer conjugate containing a terminal 1,1-bisphosphonate (BP) group, resulting in stealth and stable nanoparticles with enhanced properties. In vivo studies revealed a strong T1-effect, making them effective as MRI contrast

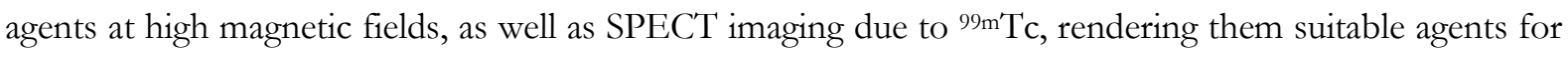
multimodal imaging [55]. For the same purpose, SPIONs were coated with dextran and radiolabeled with ${ }^{99} \mathrm{~m} T \mathrm{Tc}$ using $\mathrm{SnCl}_{2}$ as the reducing agent. The effect of different modes of administration (intravenous or intra-arterial) as well as the localization of the tumor sites with or without the addition of Lipiodol, were investigated. Finally, intra-arterially delivered complexes with Lipiodol demonstrated enhanced results against liver tumor [56]. Another dual-modality imaging agent was developed from radiolabeling iron oxide nanoparticles linked to cRGDfK-Orn3-CGG (an RGD peptide derivative) and functionalized with 3aminopropyltriethoxysilane (APTES), for targeting U87MG glioblastoma cells in animal models. The radiolabeled construct was proved to be a highly stable complex in vitro, which had the ability to reach satisfying ablative temperatures in vivo. These RGD-targeted nanoparticles demonstrated enhanced accumulation in tumor sites with negligible accumulation in other organs [57]. The properties of magnetic and gold nanoparticles, once combined, could lead to enhanced dual-modality probes. This was the basic idea behind the work of Felber et al (2015), who developed gold containing magnetite $\left(\mathrm{Fe}_{3} \mathrm{O}_{4}\right)$ nanoparticles. The metal surface consisted of an anchor for Au, bifunctional ligands and chelating agents for the $\left[{ }^{99 \mathrm{~m} T c}(\mathrm{CO})_{3}\right]^{+}$moiety. The synthesis was approached in two different pathways, both of which showed promising results [58].

Silica nanoparticles could prove to be an efficient platform both for dual-imaging applications and targeted delivery of anti-tumor agents. This type of nanosystem was developed when polyamidoamine (PAMAM) was grafted onto silica nanoparticles, which were thereafter conjugated with fluorescent dye (indocyanine green (ICG)), loaded with ${ }^{99 \mathrm{~m}} \mathrm{Tc}$ and functionalized with an anti-HER 2 antibody, which provided sufficient targeting capabilities as shown by in vitro and in vivo studies [59]. An effort was made to develop radiolabeled mesoporous silica nanoparticles (MSNs) as imaging agents, with the functionalization of the 
nanoparticles with 3-aminopropyltriethoxysilane (APTES) and diethylenetriaminepentaacetic acid (DTPA). Results demonstrated the expected high uptake by liver and especially high specificity for the lung. Given the fact that MSNs are able to carry a significant drug load at the same time, they could also be potential theranostic agents [60]. A structure consisting of manganese oxide $\left(\mathrm{MnO}_{\mathrm{x}}\right)$ within the mesopores of MSNs, which was also loaded with doxorubicin, was designed and evaluated as a probe for both hybrid SPECTMR imaging but also as a therapeutic tool [61]. In another study based on MSNs, two strategies were tested. In the first strategy, safranin $\mathrm{O}$ was loaded onto the pores of the nanoparticles and the MSNs were functionalized with APTES. Then they proceeded to the addition of MUC1 aptamer, which enables targeted delivery to MDA-MB-231 cells. The second strategy was on the same track as the one mentioned before, only doxorubicin was used instead of safranin $\mathrm{O}$, with considerably increased cytotoxic effects. The safranin $\mathrm{O}$ loaded complexes, were also radiolabeled with ${ }^{99 \mathrm{~m}} \mathrm{Tc}$ demonstrating enhanced specificity and internalization, after intraocular administration in MDA-MB-231 tumor-bearing mice [62].

Liposomes radiolabeled with diagnostic radioisotopes can be excellent tools for studying the pharmacokinetics of liposome-based drug delivery systems, as was the case with liposomes labeled with ${ }_{99 \mathrm{~m}} \mathrm{Tc}(\mathrm{I})(\mathrm{CO})_{3}$. Radiolabeling techniques and their effect on the biodistribution were explored, namely direct labeling via a carboxyl group and chelation via a pyridyl ethyl cysteine compound. It was shown that both systems exhibited passive tumor accumulation [63].

Self-assembled nanoparticles were prepared with chitosan and folated poly- $\gamma$-glutamic acid and directly

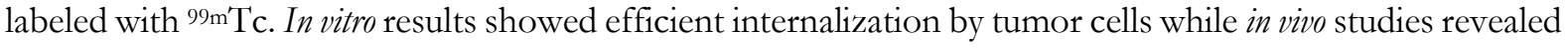
improved uptake in the tumorous kidney when compared to the normal one, as well as enhanced contrast in SPECT and SPECT/CT tumor imaging [64]. Dendrimers were synthesized, functionalized with folic acid and radiolabeled with ${ }^{99 \mathrm{~m} T c}$ via a chelating agent by Zhang et al (2010) with a radiochemical yield up to $98.9 \%$, excellent in vitro and in vivo stability, rapid blood clearance and specific tumor accumulation [65]. The same group next reported on the synthesis of a dendrimer initially reacting with biotin, which was further reacted with avidin. Both resulting species were efficiently radiolabeled with ${ }^{99 \mathrm{~m}} \mathrm{Tc}$ and comparatively evaluated in vitro in HeLa cells. As the cellular uptake was much higher for the avidin conjugate, consequent in vitro and in vivo were performed only for this construct [66].

Generation 2 (G2) PAMAM dendrimer-entrapped gold nanoparticles, radiolabeled via chelation and modified with folic acid (FA) or RGD peptide, were devised by Li et al (2016) and Xu X. et al (2017) respectively [67], [68]. The chelating agents used in each study were the linear chelate DTPA and and the cyclic chelate NOTA, which were conjugated onto the surface of the dendrimers, enabling ${ }^{99 \mathrm{~m} T c}$ to bind, thus rendering both nanoprobes suitable for SPECT and CT imaging.

\subsection{Indium-111}

In a facile and robust method developed by Laan et al (2016), tropolone and ${ }^{111} \mathrm{In}$ were entrapped in the core of micelles, without the surface conjugation of the chelator that might lead to altered biological behavior and compromised stability. Ex vivo biodistribution studies in healthy mice demonstrated high liver and spleen accumulation and significant blood circulation even at 24h post-injection [69]. Micelles crosslinked with pluronic unimers and more specifically poly(ethylene oxide) and poly(propylene oxide) (PEOPPO-PEO), and radiolabeled with ${ }^{111}$ In via DTPA, were studied with respect to in vivo biodistribution using SPECT. The effecr of PEO block length on renal clearance and overall liver uptake, as well as the influence of the aggregation state on long term accumulation in the liver, were investigated [70].

Gold nanoparticles have been used for the molecular targeting of low- and high- $\alpha_{\nu} \beta_{3}$ integrin-expressing tumors. Although the radiolabeling with ${ }^{111}$ In was achieved without surface functionalization with metal chelators, biodistribution and imaging studies demonstrated a stable radiolabeled nanoparticle [71]. The tumoricidal properties of gold nanoparticles after coating with PEG, further linked to trastuzumab and ${ }^{111}$ In via DTPA chelation, were investigated. Intratumoral administration in overexpressing HER2-positive breast cancer animal models demonstrated efficient internalization, halting tumor growth as well as inducing DNA double strand breaks (DSB), thereby reducing the surviving fraction of cancer cells [72]. Song L. et al 
(2016) proposed an easy protocol to radiolabel Au nanoparticles targeting epidermal growth factor receptor (EGFR) positive tumors ${ }^{111}$ In with via the DTPA chelator [73].

Superparamagnetic iron oxide nanoparticles were used for the development of bioprobes for early diagnosis of cancer. Conjugation with ${ }^{111}$ In-labeled antimesothelin antibody $\mathrm{mAbMB}$ provided the SPIONs with the capacity to localize and image mesothelin-expressing cancer cells with both SPECT and MR imaging modalities [74]. Zolata H. et al (2015) decorated the surface of SPIONs with APTES, PEG and then conjugated thiolated bifunctional chelator 3,6,9,15-tetraazabicyclo[9.3.1]pentadeca-1(15),11,13-triene3,6,9,-triacetic acid (PCTA). Furthermore, the nanoplatform was actively targeted with the monoclonal antibody trastuzumab, and drug-loaded with doxorubicin. Further studies proved their accumulation in tumors due to the EPR effect and HER2 receptor targeting, as well as their efficacy as therapeutic agents in breast cancer animal models [75]. In order to exploit the advantages provided by triple-modal imaging, Bai J. et al (2016) designed magnetic nanocapsules (NCs) loaded with hydrophobic SPIONs. These spherical NCs were shown to be capable of fluorescent imaging (by using indocyanine green), MRI (due to the SPIONs) as well as nuclear imaging (due to ${ }^{111}$ In labeling). Studies performed in tumor-bearing mice demonstrated increased tumor uptake after application of an external magnet at the tumor area, in comparison to NCs delivery to the tumor site via EPR [76].

A copolymer consisting of poly(lactic acid) (PLA) and PEG, radiolabeled with ${ }^{111}$ In was investigated by Banerjee S.R et al (2017). SPECT and Near-infrared fluorescence (NIFR) imaging were used to reveal its pharmacokinetics and biodistribution, providing a direct comparison between the PSMA-targeted and the non-targeted complex. Similar accumulation in all tissues was demonstrated by both complexes except in tumor and liver, where they demonstrated different uptake and stability results. Microscopy studies showed that accumulation of the non- PSMA-targeted nanoparticles was less epithelium-specific, and dependent on an EPR and phagocytosis combination [77].

\subsection{Iodine-125, 131}

A method that enables rapid radiolabeling with ${ }^{125} \mathrm{I}$ of silver nanoparticles capable of tracking the in vivo tissue uptake after their systemic administration, was developed by Chrastina A. and Schnitzer J.E. (2010). Nanoparticles were labeled via chemisorption of ${ }^{125}$ I onto the Ag surface and administered intravenously. Biodistribution studies and SPECT imaging demonstrated high uptake and accumulation in the liver and spleen, something that should be investigated as it could lead to potential toxicity issues [78]. Seven years later, another simple and concomitantly efficient method was introduced for radiolabeling three types of Ag nanoparticles; pure Ag, a polyvidone (PVP)-Ag complex and a doxorubicin-PVP-Ag NP conjugate. Biodistribution studies revealed higher uptake in cancerous cells for the latter nanocomplex, rendering it as a promising theranostic agent [79].

A dual-modality imaging agent applicable in SPECT and MRI was developed by modifying USPIOs with an RGD peptidex. Radiolabeled nanoparticles with high radiochemical purity, stability and tumor specificity were produced, with biodistribution and in vivo studies verifying the promising abilities of these nanoconstructs as radiotracers of $a_{v} \beta_{3}$-overexpressing tumors. Replacement of ${ }^{125} \mathrm{I}$ with ${ }^{123} /{ }^{124} \mathrm{I}$ or ${ }^{131} \mathrm{I}$ could provide a PET radiotracer or therapeutic agent, respectively [80]. Another similar agent was constructed by Wang J. et al (2016) who conjugated ${ }^{125} \mathrm{I}-\mathrm{c}(\mathrm{RGDyK})$ peptide onto magnetic nanoparticles that had first been coated with PEG. This construct targeted U87MG tumors in murine models, which were in turn treated with photothermal therapy in vivo. SPECT/MR imaging modalities confirmed low mononuclear phagocyte uptake [81]. Radiolabeled hydroxyapatite (HAp) nanoparticles with and without further modification were investigated, pertaining to their capability as organ-targeting carriers. The nanosystems evaluated were radiolabeled with ${ }^{125} \mathrm{I}$ and consisted of HAp nanoparticles, HAp modified with chitosan (Ch) and HAp nanoparticles with a coating of blended $\mathrm{Ch}$ with poly-D,L-lactide-co-glycolide polymer (PLGA). The stability, kinetics and targeting behavior of these three constructs were evaluated in vitro and in vivo, and demonstrated significant differences from one group of nanoparticles to the other, with both the chitosan coated nanoparticles exhibiting elevated radioactivity in various organs [82]. 
Recently, Clanton R. et al (2018), exploited the strong interactions developed between gold and iodine atoms, and proceeded to synthesize Au nanocarriers with radioactive ${ }^{125}$ I integrated into their structure. The timing of ${ }^{125} \mathrm{I}$ addition was investigated, as it is blamed for the aggregation that occurs when it is added too early in the synthetic process. As AuNPs target neoplastic cells with increased energy consumption, these stable ${ }^{125}$ I-labeled AuNPs show great potential as theranostic agents (x-ray/CT imaging due to the AuNPs and therapy due to ${ }^{125} \mathrm{I}$ [83]. A variety of metal-organic complexes was investigated, focusing especially on Cobalt (Co) nanotubes (NTs) functionalized with folic acid. Doxorubicin was embedded in these targeted nanocarriers which were traceable in murine models with the aid of ${ }^{131}$ I. Tumor growth was found to be suspended in vivo, with minimal side effects reported [84].

A self-assembled amphiphilic, protein-based conjugate comprised of hydrophobic maleimidefunctionalized poly-( $\varepsilon$-caprolactone) (PCL) covalently linked to hydrophilic bovine serum albumin (BSA), was directly labeled with ${ }^{131}$ I. After its synthesis, the BSA-PCL conjugate was functionalized with antiepidermal growth factor receptor (anti-EGFR) antibody and utilized for targeted RIT. The anti-EGFRlabeled particles exhibited higher cytotoxicity in vitro and in vivo, and higher cellular and tumor uptake in murine cancer models, when compared to particles without the antibody [85]. In a similar rationale, PEGylated liposomes linked to PCL-BSA, functionalized with an RGD peptide and labeled with ${ }^{131}$, were tested against lung cancer in NCI-H460 tumor-bearing mice, and showed great potential as cancer theranostic agents [86].

PAMAM dendrimers were synthesized, linked to HMPAO (chelator), and conjugated with Buthus martensii Karsch chlorotoxin (BmK CT) as a potential theranostic tool for glioma. Although nonradiolabeled dendrimers showed no inhibition effect on C6 glioma cells, ${ }^{131}$ I-dendrimers attained a significant decrease in the cancerous cell population [87]. Polypyrrole (PPy) nanoparticles, actively targeted with transferrin and pre-labeled with ${ }^{131} \mathrm{I}$ were synthesized using a one-step strategy for synergistic anticancer treatment, combining RIT and photothermal therapy (PTT). The biodistribution, in vivo specificity and therapeutic efficacy of these transferrin capped nanoparticles was compared to PPy@BSA${ }^{131} \mathrm{I}$, and exhibited enhanced therapeutic response after being intravenously injected in U87MG tumorbearing mice [88].

\subsection{Samarium-153}

A dual-modality imaging probe, capable of upconversion luminescence (UCL) and SPECT imaging was designed using lanthanide-based upconversion nanophosphors (UCNPs). The objective of the study was the development of a post-labeling method of the interior of these nanoparticles with ${ }^{153} \mathrm{Sm}$. It was shown that this post-labeling approach could be applicable to most of the rare earth nanoparticles for their in vivo tracking [89]. The same type of nanoparticles was studied by Cao T. et al (2013), who modified $\mathrm{NaYF}_{4}$ nanoparticles with PEG and co-doped them with $\mathrm{Yb}^{3+}, \mathrm{Er}^{3+}$ and ${ }^{153} \mathrm{Sm}^{3+}$. The ultrasmall radiolabeled nanoparticles $(<10 \mathrm{~nm})$ exhibited renal clearance and dual-modality imaging capabilities via $\gamma$-counter analysis and SPECT [90].

Hydroxyapatite nanorods (HA) incorporated with gadolinium (Gd) were post-labeled with radioactive samarium, and their biodistribution was studied. These nanosystems proved to accumulate in the liver and spleen and demonstrated difficulty in excretion from the latter. However, ${ }^{153} \mathrm{Sm}-\mathrm{HA}: \mathrm{Gd}$ showed enhanced properties in vitro and imaging abilities with both SPECT and MR imaging in vivo [91].

Another SPECT/MR imaging probe was suggested by Gholami A. and Mousavie Anijdan S.H. (2015) who synthesized SPIONs and radiolabeled them with ${ }^{153} \mathrm{Sm}$ via a DTPA dianhydride chelating agent. Biodistribution studies showed high uptake in the reticuloendothelial system (RES) while exhibiting rapid clearance from blood and other organs. Therefore, these nanoconstructs could unambiguously be considered as a useful tool in RES theranostics [92]. Samarium oxide-152 $\left.\left({ }^{152} \mathrm{Sm}\right] \mathrm{Sm}_{2} \mathrm{O}_{3}\right)$ was encapsulated in PLGA nanoparticles and activated in a nuclear reactor, thus producing radioactive $\left[{ }^{152} \mathrm{Sm}\right] \mathrm{Sm}_{2} \mathrm{O}_{3}$-PLGA NPs. A tracer kinetics study was conducted in rats, to determine the effects of different modes of administration. In the case of intravenous administration, the NPs were rapidly accumulated in the RES 
Salvanou et al., Adv. Nan. Res.; Vol. 1, Issue 1, pp: 38-55, 2018

organs, while in the case of oral administration low-dose distribution to most organs was confirmed, indicating absorption of the nanoconstructs [93].

\subsection{Holmium-166}

The theranostic capabilities of ${ }^{166} \mathrm{Ho}$, a $\beta$ - and $\gamma$ - emitting isotope, were exploited when it was mixed with SPIONs functionalized with the DTPA chelator. A product of high radiochemical purity was provided, with accumulation in the liver and spleen, proving the efficacy of these nanoconstructs as RES theranostic agents [94]. A different approach for ${ }^{166} \mathrm{Ho}$ radiolabeling was developed by Di Pasqua A.J. et al (2012) who used stable Holmium-155 in conjunction with mesoporous silica MCM-41 nanoparticles, and irradiated the system, thus producing a radioactive complex. This complex was tested in murine models with orthotopic non-small cell lung cancer, giving satisfactory results [95]. As mentioned in the example above, irradiation of a complex containing ${ }^{165} \mathrm{Ho}$ in order to acquire a radiolabeled with ${ }^{166 \mathrm{Ho}}$ carrier is a common strategy. Based on this strategy, mesoporous carbon nanoparticles (MCNs) that contain holmium oxide were neutron-activated to produce ${ }^{16}{ }^{6} \mathrm{Ho}-\mathrm{MCN}$. Consequent pegylation provided a biocompatible and readilydispersible nanoconstruct. Toxicity studies substantiated the non-toxicity of the ${ }^{165} \mathrm{Ho}$-complex towards the cancerous cells and the exact contrary effect of the radiolabeled counterpart [96].

In another study, holmium acetylacetonate was irradiated as described above, thus yielding a ${ }^{166} \mathrm{Ho}-\mathrm{labeled}$ nanodevice with sufficient radiation for nuclear imaging and radioablation of tumor sites via intratumoral injections. The great advantage of using Holmium as a contrast agent is the fact that it is a nuclide that can be used in MR, CT and SPECT imaging, as well as in therapeutic applications [97].

\subsection{Lutetium-177}

A comparative study of ${ }^{177} \mathrm{Lu}$ labeled agents was conducted by Vilchis-Juárez A. et al, who assessed the therapeutic response of 3 different complexes. The first complex consisted of radiolabeled gold nanoparticles, the second one of a radiolabeled cyclo-RGDfK $(C)$ peptide and the last one a combination of the above. Radiolabeling of all three constructs was afforded via the DOTA chelator. The ${ }^{177} \mathrm{Lu}$-AuNPRGD nanoconstruct proved to be the most effective agent, demonstrating the highest uptake and retention in tumor cells, thus rendering it as a potential theranostic tool [98]. In another study, the stability of AuNPs modified with metal-chelating polymers (MCP) that embody DOTA chelators was tested. The conjugation of gold nanoparticles to MCP was afforded via a single thiol, a dithiol and a multi-thiol end group and their stability and cell uptake was evaluated [99]. The same group of Yook S. et al (2016) developed gold nanoseeds modified with PEG, radiolabeled via DOTA chelating agent and labeled with panitumumab that actively targets EGFRs, for the treatment of locally advanced breast cancer (LABC). The targeting specificity of the system was compared to the same non-targeted nanoseeds and the nanoconstructs were injected intratumorously exhibiting high radiation doses absorbed by the tumor site compared to low uptake in normal organs [100]. With the same rationale, trastuzumab-labeled nanocarriers were studied demonstrating enhanced tumor inhibition and DNA double strand breaks (DSBs) in SK-BR-3 and MDAMB-361 cancer cells, when compared to the unlabeled counterparts [101]. In yet another study, Au nanoparticles were radiolabeled with ${ }^{177} \mathrm{Lu}$ via the DOTA-Gly-Gly-Cys (DOTA-GGC) oligopeptide. These NPs were surface-functionalized with an RGD peptide and a HS-pentyl-pegaptanib aptamer, and exhibited efficient in vitro angiogenesis inhibition in the human umbilical vein cell line EA.hy926 [102].

A nanoprobe, comprised of gold nanoparticles in the cavity of PAMAM-G4 dendrimers was designed for simultaneous OI, plasmonic-PTT and targeted RIT. Bombesin and folic acid were covalently conjugated onto the dendrimers via their carboxylate groups, and were consequently radiolabeled with ${ }^{177} \mathrm{Lu}$, via the chelator S-2-[4-Isothiocyanatobenzyl]-1,4,7,10-tetraazacyclododecane tetraacetic acid (p-SCN-benzylDOTA). Results of T47D tumor cells treated with this complex showed an outstanding decrease in cell viability up to $90 \%$ [103].

A combo treatment of breast and pancreatic cancer was devised, consisting of two separate systems; an anticancer agent called cyclopamine (CPA) combined with liquid-lipid nanoparticles (LLP) and radiolabeled 
core-crosslinked polymeric micelles (CCPM). This system was proved to limit 4T1 cancer cells and inhibited tumor relapse in Miapaca-2 cell lines [104]. Satterlee A.B. et al (2015) efficiently encapsulated ${ }^{177} \mathrm{Lu}$ into lipid-calcium-phosphate (LCP) nanoparticles and studied the effect of increased radioisotope on the properties of the nanosystem. The theranostic properties of this radioisotope resulted in tumor remission in H460 and UMUC3/3T3 murine animal models, which could be visualized by SPECT and Cerenkov imaging [105].

${ }^{177} \mathrm{Lu}$-DOTATATE was encapsulated in PLGA nanoparticles coated with PEG, and were assessed regarding their pharmacokinetics and encapsulation efficacy in vitro as well as in rats in vivo. The aim of this study was to evaluate the behavior of this nanosystem as a potential peptide receptor radioisotope therapeutic (PRRT) agent [106]. Fullerene cages have also been reported as appropriate nanovehicles for radioisotope therapy by incorporating ${ }^{177} \mathrm{Lu}$. Conjugation with interleukin-13 (IL-13) peptide dyed with tetramethyl-6-carboxyrhodamine (TAMRA) was also attained, giving the possibility for targeting and imaging of glioblastoma multiforme tumors [107].

\subsection{Rhenium-186, 188}

Due to previous indications that liposomes could improve the properties of radioactive isotopes incorporated, Chen M. et al (2010) further investigated this hypothesis by producing liposomes radiolabeled with ${ }^{188}$ Re-N,N-bis(2-mercaptoethyl)-N',N'-diethylenediamine (BMEDA) and intravenously administering them in HT-29 colorectal adenocarcinoma animal models. The study of this group compared the biodistribution and imaging profiles of these liposomes with and without doxorubicin. Pharmacokinetic and bioavailability studies demonstrated similar results between the two types of liposome, which were superior to ${ }^{188} \mathrm{Re}-\mathrm{BMEDA}$ [108]. The following year, ${ }^{188} \mathrm{Re}-\mathrm{BMEDA}$ was encapsulated into liposomes, decorated with PEG and tested as a theranostic agent against brain glioma in rat models. Autoradiography, histopathological analysis and SPECT/CT imaging showed potential of this nanoconstruct and set the path for further investigation [109]. A study focusing on brachytherapy of glioblastoma followed, in which liposomes were used, this time with rhenium-186 radiolabeling. Due to the short path length of ${ }^{186} \mathrm{Re}$, the behavior of this nanoconstruct was monitored with SPECT/CT imaging and gamma camera after

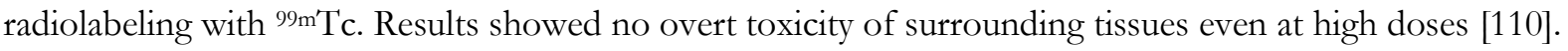
Aptes-functionalized SPIO nanoparticles were modified with the N-hydroxysuccinimide (NHS) ester of polyethylene glycol maleimide (NHS-PEG-Mal). An $\mathrm{N}_{2} \mathrm{~S}_{4}$ chelating agent was conjugated to the surface of the modified SPIONs for further radiolabeling with ${ }^{188} \mathrm{Re}$. The therapeutic and targeting efficiency of this complex was studied for both the targeted (with the addition of Rituximab) and untargeted system (for accumulation through the EPR effect). Results showed efficient tumoricidal effect of the targeted SPIONs [111].

Modification of graphene oxide nanoparticles with silica-coated, core-shell magnetic nanoparticles was attained, aiming to manufacture a peanut-shaped platform, which is supposedly more easily taken up by tumorous tissue, when compared to the spherical counterpart. These nanovehicles were loaded with gambogic acid, while polyethyleneimine (PEI) was also grafted onto their surface. Radiolabeling with ${ }^{188} \mathrm{Re}$ offered the possibility of internal radiotherapy in VX-2 tumor animal models, with simultaneous SPECT and CT imaging [112].

\subsection{Gold-198}

A decade ago, gold and dendrimer composite nanodevices (CNDs) were synthesized and radiolabeled, resulting in a poly $\left\{{ }^{\{198} \mathrm{Au}^{0}\right\} \mathrm{CND}$ system targeting melanoma tumors which exhibited up to $45 \%$ regression in murine models [113]. A couple of years later, gold nanoparticles labeled with ${ }^{198} \mathrm{Au}$ and functionalized with gum arabic glycoprotein (GA) were administered intratumorally and studied in vivo, demonstrating high tumor affinity in human prostate tumor-bearing mice. Clinical studies showed surpassing tumor volume reduction up to $82 \%$ comparing to the control group, and pharmacokinetics of the nanosystem substantiated minimal or no leakage of radioactivity to the non-targeted organs [114]. On the same pathway, 
Salvanou et al., Adv. Nan. Res.; Vol. 1, Issue 1, pp: 38-55, 2018

gold nanoparticles were also functionalized with epigallocatechin-gallate (EGCg) and radiolabeled in order exploit the strong antioxidant properties of EGCg. Besides its antioxidant properties, EGCg targets the laminin receptor Lam67R, which is overexpressed on human prostate cancer cells, resulting in similar tumor volume reduction as mentioned above [115]. Since studies with ${ }^{198} \mathrm{Au}$ focus on gold nanoparticles, a comparison study of similar-sized but differently shaped (spheres, disks, rods, cages) PEGylated gold nanoparticles was conducted in EMT6 tumors. The results of this study demonstrated enhanced accumulation of the nanospheres and nanodisks in tumorous sites albeit on the surface, when compared to nanorods and nanocages which presented a lower accumulation but better intratumoral distribution [116]. The nascent possibilities of ${ }^{198} \mathrm{Au}$-doped gold nanocages were reported by Wang Y. et al (2013), who demonstrated the unprecedented Cerenkov luminescent capabilities of the radioisotope. The radiolabeled nanostructures were decorated with PEG chains and offered luminescent imaging of EMT-6 tumors after intravenous administration in murine animal models [117]. In an effort to incorporate radioisotopic ${ }^{199} \mathrm{Au}$ in Au NPs, Zhao Y. et al (2016) once again used gold nanoparticles as nanocarriers. PEGylation and linking to DAPTA followed, in order to provide efficient imaging via SPECT of a triple negative breast cancer (TNBC) and its metastasis [118].

\subsection{Bismuth-213}

A liposomal carrier for ${ }^{213} \mathrm{Bi}$ was designed by Lingappa M. et al (2010), who functionalized the liposome with DTPA for radiolabeling, and with a mouse anti-Her/neu monoclonal antibody for targeted treatment of metastatic mammary carcinoma. The targeted liposomal vehicle, when compared to the same construct without the antibody and to the radiolabeled antibody without the liposome, showed increased cytotoxicity when compared to the non-targeted construct, and less efficacy when compared to the radiolabeled antibody [119].

\subsection{Radium-223,225}

With alpha decay nanoparticles, a major challenge is faced, namely the inability of traditional chelating agents to retain the radioactive daughters of the parent radioisotope at the targeting site without damaging adjacent tissue. For this purpose, core lanthanum phosphate and core-shell (with up to two shells) nanoparticles were evaluated pertaining to their capability to retain ${ }^{223} \mathrm{Ra}$ and ${ }^{225} \mathrm{Ra} /{ }^{225} \mathrm{Ac}$ isotopes, and were shown to exhibit satisfactory retention abilities as radioisotope nanocarriers [120].

Mokhodoeva et al investigated the direct labeling of SPIONs with ${ }^{223} \mathrm{Ra}$, the first alpha-emitting radioisotope with FDA approval for clinical application. It was demonstrated that the $\left[{ }^{223} \mathrm{Ra}\right] \mathrm{Fe}_{3} \mathrm{O}_{4} \mathrm{NPs}$ retained their radiolabel for up to $24 \mathrm{~h}$ post-preparation, while in vitro stability studies in PBS, bovine plasma and serum at 11.4 and 22.8 days showed a maximum average ${ }^{223} \mathrm{Ra}$ release of $5 \%$, rendering these NPs suitable for further in vivo testing [121]. The group of Piotrowska A. et al (2017) utilized type A nanozeolite nanoparticles containing sodium, which were linked to an NK-1 receptor-targeting peptide. These NPs were pegylated and labeled with ${ }^{223} \mathrm{Ra}$ by ion exchange with the sodium. Results showed efficient targeting of glioma cells and satisfactory retention of the radioisotope in the NPs [122].

\subsection{Actinium-225}

A multi-shell nanosystem consisting of lanthanide phosphate core-covered with a gold shell and four additional shells of gadolinium phosphate was devised in order to overcome the difficulties reported on targeted alpha therapy (TAT) [123]. The same group proceeded to further functionalize these nanoparticles with the monoclonal antibody 201b via the carboxylate group of discrete polyethylene glycol chains. Tumor-eradication properties of the nanoconstruct were demonstrated, with the daughter isotope ${ }^{213} \mathrm{Bi}$ being retained by the nanoparticles in the lung up to $\sim 90 \%$ at $24 \mathrm{~h}$ post-injection [124]. 


\section{Conclusions}

Despite the numerous successful studies of radiolabeled nanoparticles applied in adjuvant therapy, work in this field is still in its infancy and there is a need for research that performs in-depth investigation on real diagnostic or therapeutic applications with potential for clinical translation. It is expected that improved NPs will be developed, based on the accumulated knowledge and technology in which radioisotopes promise to continue playing an important role. We hope that these multifunctional NPs for multimodal imaging and theranostics can overcome present challenges and provide great contributions to human health in the near future.

\section{How to Cite this Article:}

E. Salvanou, P. Bouziotis, and C. Tsoukalas, "Radiolabeled Nanoparticles in Nuclear Oncology", Advanced Nano Research, vol. 1, no. 1, pp. 38-55, Apr. 2018. doi: 10.21467/anr.1.1.38-55

\section{References}

[1] World Health Organization, "The top 10 causes of death," www.who.int/mediacentre/factsheets/fs310/en/, 2017. .

[2] J. Ferlay et al., "GLOBOCAN 2012 v1.0, Cancer Incidence and Mortality Worldwide: IARC CancerBase No. 11," Lyon, France, 2013.

[3] R. Sharma et al., "Carbon-11 radiolabeling of iron-oxide nanoparticles for dual-modality PET/MR imaging," Nanoscale, vol. 5, no. 16, pp. 7476-7483, 2013.

[4] M. Jauregui-osoro, P. A. Williamson, A. Glaria, K. Sunassee, P. Charoephun, and M. A. Green, "Biocompatible inorganic nanoparticles for [18F]-fluoride binding with applications in PET imaging," Dalt. Trans., vol. 40, no. 23, pp. 6226-6237, 2011.

[5] S. Guerrero et al., "Synthesis and In Vivo Evaluation of the Biodistribution of a 18 F- Labeled Conjugate Gold-Nanoparticle-Peptide with Potential Biomedical Application," Bioconjug. Chem., vol. 23, pp. 399-408, 2012.

[6] Z. Sun et al., "Robust surface coating for a fast, facile fluorine-18 labeling of iron oxide nanoparticles for PET/MR dual-modality imaging," Nanoscale, vol. 8, no. 47, pp. 19644-19653, 2016.

[7] F. Emmetiere et al., "F-labeled-Bioorthogonal Liposomes for In Vivo Targeting," Bioconjug. Chem., vol. 24, no. 11, pp. 17841789, 2014.

[8] P. P. Di Mauro, V. Gómez-Vallejo, Z. Baz Maldonado, J. Llop Roig, and S. Borrós, "Novel 18F Labeling Strategy for PolyesterBased NPs for in Vivo PET-CT Imaging," Bioconjug. Chem., vol. 26, no. 3, pp. 582-592, 2015.

[9] S. Berke et al., "18F-Radiolabeling and in Vivo Analysis of SiFA-Derivatized Polymeric Core-Shell Nanoparticles," Bioconjug. Chem., vol. 29, no. 1, pp. 89-95, 2018.

[10] S. Lee et al., "Copper-64 labeled liposomes for imaging bone marrow," Nucl. Med. Biol., vol. 43, no. 12, pp. 781-787, 2016.

[11] H. Lee et al., "A gradient-loadable $64 \mathrm{Cu}$-chelator for quantifying tumor deposition kinetics of nanoliposomal therapeutics by positron emission tomography," Nanomedicine Nanotechnology, Biol. Med., vol. 11, no. 1, pp. 155-165, 2015.

[12] Y. Du et al., "Nuclear and Fluorescent Labeled PD-1-Liposome-DOX-64Cu/IRDye800CW Allows Improved Breast Tumor Targeted Imaging and Therapy," Mol. Pharm., vol. 14, no. 11, pp. 3978-3986, 2017.

[13] P. Wong et al., "PET imaging of64Cu-DOTA-scFv-anti-PSMA lipid nanoparticles (LNPs): Enhanced tumor targeting over antiPSMA scFv or untargeted LNPs," Nucl. Med. Biol., vol. 47, pp. 62-68, 2017.

[14] B. Pang et al., "64Cu-Doped PdCu@ Au Tripods: A Multifunctional Nanomaterial for Positron Emission Tomography and ImageGuided Photothermal Cancer Treatment," ACS Nano, vol. 10, no. 3, pp. 3121-3131, 2016.

[15] A. F. Frellsen et al., "Mouse Positron Emission Tomography Study of the Biodistribution of Gold Nanoparticles with Different Surface Coatings Using Embedded Copper-64," ACS Nano, vol. 10, pp. 9887-9898, 2016.

[16] X. Tong et al., "Size dependent kinetics of gold nanorods in EPR mediated tumor delivery," Theranostics, vol. 6, no. 12, pp. 20392051, 2016.

[17] Y. Zhao et al., "Gold Nanoclusters-Doped With 64Cu for CXCR4 Positron Emission Tomography Imaging of Breast Cancer and Metastasis," ACS Nano, vol. 10, no. 6, pp. 5959-5970, 2016.

[18] D. Lee et al., "Facile Method To Radiolabel Glycol Chitosan Nanoparticles with $64 \mathrm{Cu}$ via Copper-Free Click Chemistry for MicroPET Imaging," Mol. Pharm., vol. 10, pp. 2190-2198, 2013.

[19] T. M. Shaffer, S. Harmsen, E. Khwaja, M. F. Kircher, C. M. Drain, and J. Grimm, "Stable Radiolabeling of Sulfur-Functionalized Silica Nanoparticles with Copper-64," Nano Lett., vol. 16, pp. 5601-5604, 2016.

[20] R. Torres Martin de Rosales et al., "Synthesis of ${ }^{64} \mathrm{Cu}{ }^{\text {II }}$-Bis(dithiocarbamatebisphosphonate) and Its Conjugation with Superparamagnetic Iron Oxide Nanoparticles: In Vivo Evaluation as Dual-Modality PET-MRI Agent," Angew. Chemie Int. Ed., vol. 50, no. 24, pp. 5509-5513, 2011.

[21] X. Yang et al., "cRGD-functionalized, DOX-conjugated, and 64Cu-labeled superparamagnetic iron oxide nanoparticles for targeted anticancer drug delivery and PET/MR imaging," Biomaterials, vol. 32, pp. 4151-4160, 2011.

[22] D. Yang et al., "In vivo targeting of metastatic breast cancer via tumor vasculature-specific nano-graphene oxide," Biomaterials, vol. 104, pp. 361-371, 2016.

[23] Y. Zhan et al., "Radiolabeled, Antibody-Conjugated Manganese Oxide Nanoparticles for Tumor Vasculature Targeted Positron Emission Tomography and Magnetic Resonance Imaging,” ACS Appl. Mater. Interfaces, vol. 9, no. 44, pp. 38304-38312, 2017.

[24] C. Truillet et al., "Ultrasmall particles for Gd-MRI and68Ga-PET dual imaging," Contrast Media Mol. Imaging, vol. 10, no. 4, pp. 309-319, 2015. 
Salvanou et al., Adv. Nan. Res.; Vol. 1, Issue 1, pp: 38-55, 2018

[25] P. Bouziotis et al., "Ga-radiolabeled AGuIX nanoparticles as dual-modality imaging agents for PET / MRI- guided radiation therapy," Nanomedicine, vol. 12, no. 13, pp. 1561-1574, 2017.

[26] J. Frigell, I. García, V. Gómez-Vallejo, and J. Llop, "Ga-Labeled Gold Glyconanoparticles for Exploring Blood - Brain Barrier Permeability: Preparation, Biodistribution Studies, and Improved Brain Uptake via Neuropeptide Conjugation,” J. Am. Chem. Soc., vol. 136, pp. 449-457, 2014.

[27] B. P. Burke et al., "Final step gallium-68 radiolabelling of silica-coated iron oxide nanorods as potential PET/MR multimodal imaging agents," Faraday Discuss., vol. 175, pp. 59-71, 2014.

[28] S. Moon et al., "Development of a complementary PET / MR dual-modal imaging probe for targeting prostate-specific membrane antigen ( PSMA )," Nanomedicine Nanotechnology, Biol. Med., vol. 12, no. 4, pp. 871-879, 2016.

[29] B. Y. Yang, S.-H. Moon, S. R. Seelam, M. J. Jeon, and Y.-S. Lee, "Development of a multimodal imaging probe by encapsulating iron oxide nanoparticles with functionalized amphiphiles for lymph node imaging," Nanomedicine, vol. 10, no. 12, pp. 1899-1910, 2015.

[30] Y. Fazaeli, R. Rahighi, A. Tayyebi, and S. Feizi, "Synthesis, characterization and biological evaluation of a well dispersed suspension of gallium-68-labeled magnetic nanosheets of graphene oxide for in vivo coincidence imaging," Radiochim. Acta, vol. aop, pp. 1-9, 2016.

[31] A. Polyak et al., "Journal of Pharmaceutical and Biomedical Analysis Preparation and 68 Ga-radiolabeling of porous zirconia nanoparticle platform for PET / CT-imaging guided drug delivery," J. Pharm. Biomed. Anal., vol. 137, pp. 146-150, 2017.

[32] M. Jennewein et al., "A new method for radiochemical separation of arsenic from irradiated germanium oxide," Appl. Radiat. Isot., vol. 63, no. 3, pp. 343-351, 2005.

[33] F. Chen et al., "Chelator-Free Synthesis of a Dual-Modality PET / MRI Agent ** Angewandte," Angew. Chemie - Int. Ed., vol. 125, pp. 13561-13565, 2013.

[34] L. Karmani et al., "Antibody-functionalized nanoparticles for imaging cancer : influence of conjugation to gold nanoparticles on the biodistribution of 89 Zr-labeled cetuximab in mice," Contrast Media Mol. Imaging, vol. 8, no. February, pp. 402-408, 2013.

[35] E. Boros, A. M. Bowen, L. Josephson, and J. P. Holland, "Chelate-free metal ion binding and heat-induced radiolabeling of iron oxide nanoparticles," Chem. Sci., vol. 6, pp. 225-236, 2015.

[36] D. L. J. Thorek et al., "Non-invasive mapping of deep-tissue lymph nodes in live animals using a multimodal PET/MRI nanoparticle," Nat. Commun., vol. 5, p. 3097, 2014.

[37] L. Cheng et al., "Chelator-Free Labeling of Metal Oxide Nanostructures with Zirconium-89 for Positron Emission Tomography Imaging," ACS Nano, vol. 11, no. 12, pp. 12193-12201, 2017.

[38] D. Chen et al., "In Vivo Targeting and Positron Emission Tomography Imaging of Tumor with Intrinsically Radioactive MetalOrganic Frameworks Nanomaterials," ACS Nano, vol. 11, no. 4, pp. 4315-4327, 2017.

[39] F. Chen et al., "In Vivo Integrity and Biological Fate Mesoporous Silica Nanoparticles," ACS Nano, vol. 9, no. 8, pp. 7950-7959, 2015.

[40] N. Li, Z. Yu, T. T. Pham, and E. Al, "A generic $89 \mathrm{Zr}$ labeling method to quantify the in vivo pharmacokinetics of liposomal nanoparticles with positron emission tomography," Int. J. Nanomedicine, vol. 12, pp. 3281-3294, 2017.

[41] C. Truillet, E. Thomas, F. Lux, L. T. Huynh, O. Tillement, and M. J. Evans, "Synthesis and characterization of 89Zr-labeled ultrasmall nanoparticles Synthesis and characterization of 89 Zr-labeled ultrasmall nanoparticles," Mol. Pharm., vol. 13, no. 7, pp. 2596-2601, 2016.

[42] H. Groult et al., "Parallel multifunctionalization of nanoparticles: A one-step modular approach for in vivo imaging," Bioconjug. Chem., vol. 26, pp. 153-160, 2015.

[43] M. Radovic, M. P. Calatayud, G. F. Goya, M. R. Ibarra, and B. Antic, "Preparation and in vivo evaluation of multifunctional 90Ylabeled magnetic nanoparticles designed for cancer therapy," J. Biomed. Mater. Res., vol. 103, no. 1, pp. 126-134, 2015.

[44] M. Radovic et al., "Development and evaluation of 90 Y-labeled albumin microspheres loaded with magnetite nanoparticles for possible applications in cancer therapy," J. Mater. Chem., vol. 22, pp. 24017-24025, 2012.

[45] A. Kursad, A. Yenilmez, and H. Eroglu, "Evaluation of radiolabeled curcumin-loaded solid lipid nanoparticles usage as an imaging agent in liver-spleen scintigraphy,” Mater. Sci. Eng. C, vol. 75, pp. 663-670, 2017.

[46] E. Ucar et al., "Synthesis, characterization and radiolabeling of folic acid modified nanostructured lipid carriers as a contrast agent and drug delivery system," Appl. Radiat. Isot., vol. 119, pp. 72-79, 2017.

[47] K. K. Halder, B. Mandal, M. C. Debnath, H. Bera, L. K. Ghosh, and B. K. Gupta, "Chloramphenicol-incorporated poly lactide- co -glycolide ( PLGA ) nanoparticles : Formulation , characterization , technetium-99m labeling and biodistribution studies," J. Drug Target., vol. 16, no. 4, pp. 311-320, 2008.

[48] S. Subramanian, P. Dandekar, R. Jain, U. Pandey, and G. Samuel, "Nanoparticles as an Alternative," Cancer Biotherary and Radioplarmaceuticals, vol. 25, no. 6, pp. 637-644, 2010.

[49] S. Subramanian, U. Pandey, D. Gugulothu, V. Patravale, and G. Samuel, "Modification of PLGA Nanoparticles for Improved Properties as a 99m Tc-Labeled Agent in Sentinel Lymph Node Detection," Cancer Biotherary and Radioplarmaceuticals, vol. 28, no. 8, pp. 598-606, 2013.

[50] B. F. De Carvalho Patricio, M. De Souza Albernaz, M. A. Sarcinelli, S. M. De Carvalho, R. Santos-Oliveira, and G. Weissmüller, "Development of novel nanoparticle for bone cancer," J. Biomed. Nanotechnol., vol. 10, no. 7, pp. 1242-1248, 2014.

[51] B. E. Ocampo-garcía et al., "Tc-labelled gold nanoparticles capped with HYNIC-peptide / mannose for sentinel lymph node detection 论," Nucl. Med. Biol., vol. 38, no. 1, pp. 1-11, 2011.

[52] A. N. Mendoza-sánchez et al., "Lys 3 -Bombesin Conjugated to 99m Tc-Labelled Gold Nanoparticles for In Vivo Gastrin Releasing Peptide-Receptor Imaging,” vol. 6, no. 4, pp. 375-384, 2010.

[53] G. Ferro-flores, E. Morales-avila, and F. De Mar1, "Kit for preparation of multimeric receptor-specific Tc-radiopharmaceuticals based on gold nanoparticles Blanca Ocampo-Garc1," 2011.

[54] L. Zhao et al., "“99m Tc-labelled multifunctional polyethylenimine-entrapped gold nanoparticles for dual mode SPECT and CT 
imaging," Artif. Cells, Nanomedicine Biotechnol., vol. 1401, 2018.

[55] T. M. Imaging, M. Botnar, P. J. Blower, G. Frodsham, P. A. Williamson, and N. Gaddum, "Terms of Use Bisphosphonate-Anchored PEGylation and Radiolabeling of Superparamagnetic Iron Oxide : Long-Circulating Nanoparticles for in Vivo Multimodal," ACS Nano, vol. 7, no. 1, pp. 500-512, 2013.

[56] I. J. Lee et al., "Image-based analysis of tumor localization after intra-arterial delivery of technetium-99m-labeled SPIO using SPECT/CT and MRI," Mol. Imaging, vol. 16, pp. 1-9, 2017.

[57] I. Tsiapa et al., "99mTc-labeled aminosilane-coated iron oxide nanoparticles for molecular imaging of $\alpha v \beta 3$-mediated tumor expression and feasibility for hyperthermia treatment," J. Colloid Interface Sci., vol. 433, pp. 163-175, 2014.

[58] A. F. O, M. Felber, and R. Alberto, "99mTc radiolabelling of Fe3O4-Au core-shell and Au-Fe3O4 dumbell-like nanoparticles," Nanoscale, vol. 7, pp. 6653-6660, 2015.

[59] H. Yamaguchi, M. Tsuchimochi, K. Hayama, and T. Kawase, "Dual-Labeled Near-Infrared / 99m Tc Imaging Probes Using PAMAM-Coated Silica Nanoparticles for the Imaging of HER2-Expressing Cancer Cells," Int. J. Mol. Sci., vol. 17, p. 1086, 2016.

[60] A. Luís et al., "Synthesis, characterization, and biodistribution studies of 99m Tc-labeled SBA-16 mesoporous silica nanoparticles," Mater. Sci. Eng. C, vol. 56, pp. 181-188, 2015.

[61] H. Gao et al., "99mTc-conjugated manganese-based mesoporous silica nanoparticles for SPECT, pH-responsive MRI and anticancer drug delivery," Nanoscale, vol. 8, no. 47, pp. 19573-19580, 2016.

[62] L. Pascual et al., "MUC1 aptamer-capped mesoporous silica nanoparticles for controlled drug delivery and radio-imaging applications," Nanomedicine Nanotechnology, Biol. Med., vol. 13, pp. 2495-2505, 2017.

[63] G. Fragogeorgi, E.A., Savina, I.N., Tsotakos, T., Efthimiadou, E., Xanthopoulos, S., Palamaris, L., Psimadas, D., Bouziotis, P., Kordas, G., Mikhalovsky, S., Alavijeh, M., and Loudos, "Comparative in vitro stability and scintigraphic imaging for trafficking and tumor targeting of a directly and a novel 99mTc (I)(CO)3 labeled liposome.," Int. J. Pharm., vol. 465(1-2), no. I, pp. 333-346, 2014.

[64] A. Polyák, I. Hajdu, M. Bodnár, G. Trencsényi, and Z. Pöstényi, “Tc-labelled nanosystem as tumour imaging agent for SPECT and SPECT / CT modalities,” Int. J. Pharm., vol. 449, pp. 10-17, 2013.

[65] Y. Zhang, Y. Sun, X. Xu, H. Zhu, L. Huang, and X. Zhang, "Bioorganic \& Medicinal Chemistry Letters Radiosynthesis and microSPECT imaging of poly ( amido ) -amine folic acid conjugate Tc-dendrimer,” Bioorg. Med. Chem. Lett., vol. 20, no. 3, pp. 927931,2010

[66] X. Xu et al., "Bioorganic \& Medicinal Chemistry Radiosynthesis, biodistribution and micro-SPECT imaging study of dendrimer avidin conjugate," Bioorg. Med. Chem., vol. 19, no. 5, pp. 1643-1648, 2011.

[67] X. Li et al., "99mTc-Labeled Multifunctional Low Generation Dendrimer-Entrapped Gold Nanoparticles for Targeted SPECT / CT Dual-Mode Imaging of Tumors Tc-Labeled Multifunctional Low Generation Dendrimer-Entrapped Gold Nanoparticles for Targeted SPECT / CT Dual-Mode Imag,” ACS Appl. Mater. Interfaces, vol. 8, no. 31, pp. 19883-19891, 2016.

[68] X. Xu et al., "Targeted tumor SPECT/CT dual mode imaging using multifunctional RGD-modified low generation dendrimerentrapped gold nanoparticles," Biomater. Sci., vol. 5, no. 12, 2017.

[69] A. C. Laan, C. Santini, L. Jennings, M. De Jong, M. R. Bernsen, and A. G. Denkova, "Radiolabeling polymeric micelles for in vivo evaluation : a novel, fast, and facile method," EJNMMI Res., vol. 6, p. 12, 2016.

[70] A. Arranja et al., "SPECT/CT Imaging of Pluronic Nanocarriers with Varying Poly(ethylene oxide) Block Length and Aggregation State," Mol. Pharm., vol. 13, pp. 1158-1165, 2016.

[71] Q. K. T. Ng et al., "Biomaterials Indium-111 labeled gold nanoparticles for in-vivo molecular targeting," Biomaterials, vol. 35, pp. 7050-7057, 2014.

[72] Z. Cai et al., "In-labeled trastuzumab-modi fi ed gold nanoparticles are cytotoxic in vitro to HER2-positive breast cancer cells and arrest tumor growth in vivo in athymic mice after intratumoral injection," Nucl. Med. Biol., vol. 43, no. 12, pp. 818-826, 2016.

[73] L. Song, N. Falzone, and K. A. Vallis, "EGF-coated gold nanoparticles provide an efficient nano-scale delivery system for the molecular radiotherapy of EGFR-positive cancer," Int. J. Radiat. Biol., vol. 92, no. 11, pp. 716-723, 2016.

[74] R. Misri, D. Meier, A. C. Yung, P. Kozlowski, and U. O. Häfeli, "Development and evaluation of a dual-modality ( MRI / SPECT ) molecular imaging bioprobe,” Nanomedicine Nanotechnology, Biol. Med., vol. 8, pp. 1007-1016, 2012.

[75] H. Zolata, F. Abbasi, and H. Afarideh, "Synthesis, characterization and theranostic evaluation of Indium-111 labeled multifunctional superparamagnetic iron oxide nanoparticles," Nucl. Med. Biol., vol. 42, pp. 164-170, 2015.

[76] J. Bai et al., "Triple-modal imaging of magnetically-targeted nanocapsules in solid tumours in vivo," Theranostics, vol. 6, no. 3, pp. 342-356, 2016.

[77] S. R. Banerjee, C. A. Foss, A. Horhota, K. A. Mcdonnell, S. Zale, and M. G. Pomper, “An 111 In- and IRDye800CW-Labeled PLAPEG Nanoparticle for Imaging Prostate-specific membrane antigen-Expressing Tissues," Biomacromolecules, vol. 18, no. 1, pp. 201-209, 2017.

[78] A. Chrastina and J. E. Schnitzer, "Iodine-125 radiolabeling of silver nanoparticles for in vivo SPECT imaging," Int. J. Nanomedicine, vol. 5, pp. 653-659, 2010.

[79] N. S. Farrag, H. A. El-Sabagh, A. M. Al-mahallawi, A. M. Amin, A. AbdEl-Bary, and W. Mamdouh, "Comparative Study on Radiolabeling and Biodistribution of Core-shell Silver/polymeric Nanoparticles-based Theranostics for Tumor Targeting," Int. J. Pharm., vol. 521, no. 1-2, pp. 123-133, 2017.

[80] S. Deng, W. Zhang, B. Zhang, and E. Al, "Radiolabeled cyclic arginine-glycine-aspartic ( RGD ) - conjugated iron oxide nanoparticles as single-photon emission computed tomography ( SPECT ) and magnetic resonance imaging ( MRI ) dual-modality agents for imaging of breast cancer," J. Nanoparticle Res., vol. 17, p. 19, 2015.

[81] J. Wang et al., "MR / SPECT Imaging Guided Photothermal Therapy of Tumor-Targeting Fe@Fe3O4 Nanoparticles in Vivo with Low Mononuclear Phagocyte Uptake,” ACS Appl Mater Interfaces, vol. 8, no. 31, pp. 19872-19882, 2016.

[82] N. Ignjatovic, S. V. Djuric, Ž. Mitic, D. Jankovic, and D. Uskokovic, "Investigating an organ-targeting platform based on hydroxyapatite nanoparticles using a novel in situ method of radioactive 125Iodine labeling," Mater. Sci. Eng. C, vol. 43, pp. 439$446,2014$. 
Salvanou et al., Adv. Nan. Res.; Vol. 1, Issue 1, pp: 38-55, 2018

[83] R. Clanton, A. Gonzalez, S. Shankar, and G. Akabani, "Rapid synthesis of $125 \mathrm{I}$ integrated gold nanoparticles for use in combined neoplasm imaging and targeted radionuclide therapy," Appl. Radiat. Isot., vol. 131, pp. 49-57, 2018.

[84] L. X. Liu et al., "An Integrative Folate-Based Metal Complex Nanotube as a Potent Antitumor Nanomedicine as Well as an Efficient Tumor-Targeted Drug Carrier,” Bioconjug. Chem., vol. 27, no. 12, pp. 2863-2873, 2016.

[85] W. Li, Z. Liu, C. Li, N. Li, L. Fang, and J. Chang, "Radionuclide therapy using 131 I - labeled anti - epidermal growth factor receptor - targeted nanoparticles suppresses cancer cell growth caused by EGFR overexpression," J. Cancer Res. Clin. Oncol., vol. 142, no. 3, pp. 619-632, 2016.

[86] H. Ming et al., "Antitumor Effect of Nanoparticle 131 I-Labeled Arginine-Glycine-Aspartate-Bovine Serum AlbuminPolycaprolactone in Lung Cancer," Am. J. Roentgenol., vol. 208, pp. 1-11, 2017.

[87] Y. Cheng et al., "I-labeled multifunctional dendrimers modified with BmK CT for targeted SPECT imaging and radiotherapy of gliomas," Nanomedicine, vol. 11, no. 10, pp. 1253-1266, 2016.

[88] X. Song, C. Liang, L. Feng, K. Yang, and Z. Liu, "Iodine-131-labeled, transferrin-capped polypyrrole nanoparticles for tumortargeted synergistic photothermal-radioisotope therapy," Biomater. Sci., vol. 5, no. 9, pp. 1828-1835, 2017.

[89] Y. Sun et al., "Radioisotope post-labeling upconversion nanophosphors for in vivo quantitative tracking," Biomaterials, vol. 34, no. 9, pp. 2289-2295, 2013.

[90] T. Cao et al., "Biodistribution of sub-10nm PEG-modified radioactive/upconversion nanoparticles," Biomaterials, vol. 34, pp. 71277134, 2013.

[91] Y. Liu et al., "Long-term biodistribution in vivo and toxicity of radioactive/magnetic hydroxyapatite nanorods," Biomaterials, vol. 35, no. 10, pp. 3348-3355, 2014.

[92] A. Gholami and S. H. Mousavie Anijdan, "Development of 153 Sm-DTPA-SPION as a theranostic dual contrast agents in SPECT/MRI," Iran. J. Basic Med. Sci., vol. 19, pp. 1056-1062, 2016.

[93] V. Mandiwana, L. Kalombo, K. Venter, M. Sathekge, A. Grobler, and J. R. Zeevaart, "Samarium oxide as a radiotracer to evaluate the in vivo biodistribution of PLGA nanoparticles," J. Nanoparticle Res., vol. 17, p. 375, 2015.

[94] S. Nosrati, S. Shanehsazzadeh, H. Yousefnia, A. Gholami, and C. Gru, "Biodistribution evaluation of 166 Ho - DTPA - SPION in normal rats," pp. 1559-1566, 2016.

[95] A. J. Di Pasqua, M. L. Miller, X. Lu, L. Peng, and M. Jay, "Tumor accumulation of neutron-activatable holmium-containing mesoporous silica nanoparticles in an orthotopic non-small cell lung cancer mouse model," Inorganica Chim. Acta, vol. 393, pp. 334-336, 2012.

[96] J. Kim, Z. X. Luo, Y. Wu, X. Lu, and M. Jay, "In-situ formation of holmium oxide in pores of Mesoporous Carbon Nanoparticles as substrates for neutron-activatable radiotherapeutics," Carbon N. Y., vol. 117, pp. 92-99, 2017.

[97] W. Bult et al., "Holmium nanoparticles: Preparation and in vitro characterization of a new device for radioablation of solid malignancies," Pharm. Res., vol. 27, no. 10, pp. 2205-2212, 2010.

[98] A. Vilchis-Juárez, G. Ferro-Flores, C. Santos-Cuevas, E. Morales-Avila, and B. Ocampo-García, "Molecular Targeting Radiotherapy with Cyclo-RGDfK ( C ) Peptides Conjugated to 177 Lu-Labeled Gold Nanoparticles in Tumor-Bearing Mice," J. Biomed. Nanotechnol., vol. 10, no. 3, pp. 393-404, 2014.

[99] S. Yook et al., "Stability and Biodistribution of Thiol-Functionalized and 177Lu- Labeled Metal Chelating Polymers ( MCP ) Bound to Gold Nanoparticles Stability and Biodistribution of Thiol-Functionalized and Lu-Labeled Metal Chelating Polymers ( MCP ) Bound to Gold Nanop," Biomacromolecules, vol. 17, no. 4, pp. 1292-1302, 2016.

[100] S. Yook, Z. Cai, Y. Lu, M. A. Winnik, and J. Pignol, "Intratumorally Injected Lu-Labeled Gold Nanoparticles - Gold Nanoseed Brachytherapy with Application for Neo-Adjuvant Treatment of Locally Advanced Breast Cancer ( LABC )," no. 416, pp. 1-32, 2016.

[101] Z. Cai et al., "Local Radiation Treatment of HER2-Positive Breast Cancer Using Trastuzumab-Modified Gold Nanoparticles Labeled with 177Lu," Pharm. Res., vol. 34, pp. 579-590, 2017.

[102] A. González-Ruíz et al., "Synthesis and in vitro evaluation of an antiangiogenic cancer-specific dual-targeting177Lu-Aunanoradiopharmaceutical," J. Radioanal. Nucl. Chem., vol. 314, pp. 1337-1345, 2017.

[103] H. Mendoza-Nava et al., "Fluorescent, plasmonic, and radiotherapeutic properties of the177Lu-dendrimer-AuNP-folate-bombesin nanoprobe located inside cancer cells," Mol. Imaging, vol. 16, pp. 1-10, 2017.

[104] J. You et al., "Chemoradiation therapy using cyclopamine-loaded liquid-lipid nanoparticles and lutetium-177-labeled corecrosslinked polymeric micelles," J. Control. Release, vol. 202, pp. 40-48, 2015.

[105] A. B. Satterlee, H. Yuan, and L. Huang, "A radio-theranostic nanoparticle with high specific drug loading for cancer therapy and imaging," J. Control. Release, vol. 217, pp. 170-182, 2015.

[106] G. Arora, J. Shukla, S. Ghosh, S. K. Maulik, A. Malhotra, and G. Bandopadhyaya, "Plga nanoparticles for peptide receptor radionuclide therapy of neuroendocrine tumors: A novel approach towards reduction of renal radiation dose," PLoS One, vol. 7, no. 3, pp. 1-11, 2012.

[107] M. D. Shultz et al., "Encapsulation of a radiolabeled cluster inside a fullerene cage,177LuxLu(3- x)N@C80: An interleukin-13conjugated radiolabeled metallofullerene platform," J. Am. Chem. Soc., vol. 132, pp. 4980-4981, 2010.

[108] M. Chen et al., "MicroSPECT/CT Imaging and Pharmacokinetics of 188 Re-(DXR)-liposome in Human Colorectal Adenocarcinoma-bearing Mice," Anticancer Res., vol. 30, pp. 65-72, 2010.

[109] F.-Y. J. Huang et al., "Imaging, Autoradiography, and Biodistribution of ${ }^{188}$ Re-Labeled PEGylated Nanoliposome in Orthotopic Glioma Bearing Rat Model," Cancer Biother. Radiopharm., vol. 26, no. 6, pp. 717-725, 2011.

[110] W. T. Phillips et al., "Rhenium-186 liposomes as convection- enhanced nanoparticle brachytherapy for treatment of glioblastoma," Neuro. Oncol., vol. 14, no. 4, pp. 416-425, 2012.

[111] B. Azadbakht, H. Afarideh, M. Ghannadi-Maragheh, A. Bahrami-Samani, and M. Asgari, "Preparation and evaluation of APTESPEG coated iron oxide nanoparticles conjugated to rhenium-188 labeled rituximab," Nucl. Med. Biol., vol. 48, pp. 26-30, 2017.

[112] Y. Yang et al., "Rational Design of GO-Modified Fe3O4/SiO2Nanoparticles with Combined Rhenium-188 and Gambogic Acid for Magnetic Target Therapy,” ACS Appl. Mater. Interfaces, vol. 9, no. 34, pp. 28195-28208, 2017. 
Radiolabeled Nanoparticles in Nuclear Oncology

[113] M. K. Khan, L. D. Minc, S. S. Nigavekar, M. S. T. Kariapper, and B. M. Nair, "Fabrication of $\{198$ Au0 $\}$ radioactive composite nanodevices and their use for nano-brachytherapy," Nanomedicine, vol. 4, no. 1, pp. 57-69, 2008.

[114] N. Chanda et al., "Radioactive gold nanoparticles in cancer therapy: therapeutic efficacy studies of GA-198AuNP nanoconstruct in prostate tumor-bearing mice," Nanomedicine Nanotechnology, Biol. Med., vol. 6, no. 2, pp. 201-209, 2010.

[115] R. Shukla, N. Chanda, A. Zambre, A. Upendran, K. Katti, and R. R. Kulkarni, "Laminin receptor specific therapeutic gold efficacy in treating prostate cancer," PNAS, vol. 109, no. 31, pp. 12426-12431, 2012.

[116] K. C. L. Black et al., "Radioactive 198Au-Doped Nanostructures with Different Shapes for In Vivo Analyses of Their Biodistribution, Tumor Uptake, and Intratumoral Distribution," ACS Nano, vol. 8, no. 5, pp. 4385-4394, 2014.

[117] Y. Wang et al., "Radioluminescent Gold Nanocages with Controlled Radioactivity for Real-time In Vivo Imaging," Nano Lett., vol. 13, no. 2, pp. 581-585, 2013.

[118] Y. Zhao et al., "Gold Nanoparticles Doped with 199Au Atoms and Their Use for Targeted Cancer Imaging by SPECT," Adv. Healthc. Mater., vol. 5, no. 8, pp. 928-935, 2016.

[119] M. Lingappa, H. Song, S. Thompson, F. Bruchertseifer, and G. Sgouros, "Immunoliposomal Delivery of 213Bi for $\alpha$-Emitter Targeting of Metastatic Breast Cancer," Cancer Res., vol. 70, no. 17, pp. 6815-6823, 2010.

[120] J. V. Rojas, J. D. Woodward, N. Chen, A. J. Rondinone, C. H. Castano, and S. Mirzadeh, "Synthesis and characterization of lanthanum phosphate nanoparticles as carriers for 223Ra and 225Ra for targeted alpha therapy," Nucl. Med. Biol., vol. 42, no. 7, pp. 614-620, 2015.

[121] O. Mokhodoeva et al., "Study of 223Ra uptake mechanism by Fe3O4 nanoparticles: towards new prospective theranostic SPIONs," J. Nanoparticle Res., vol. 18, p. 301, 2016.

[122] A. Piotrowska et al., "Nanozeolite bioconjugates labeled with 223Ra for targeted alpha therapy," Nucl. Med. Biol., vol. 47, pp. 10$18,2017$.

[123] M. F. Mclaughlin et al., "Gold Coated Lanthanide Phosphate Nanoparticles for Targeted Alpha Generator Radiotherapy," PLoS One, vol. 8, no. 1, pp. 2-9, 2013.

[124] M. F. Mclaughlin, D. Robertson, P. H. Pevsner, and J. S. Wall, "LnPO4 Nanoparticles Doped with Ac-225 and Sequestered Daughters for Targeted Alpha Therapy," Cancer Biotherary Radiopharm., vol. 29, no. 1, pp. 34-41, 2013.

Publish your research article in AIJR journals-

$\checkmark$ Online Submission and Tracking

$\checkmark$ Peer-Reviewed

$\checkmark$ Rapid decision

$\checkmark$ Immediate Publication after acceptance

$\checkmark \quad$ Articles freely available online

$\checkmark \quad$ Retain full copyright of your article.

Submit your article at journals.aijr.in
Publish your books with AIJR publisher-

$\checkmark$ Publish with ISBN and DOI.

$\checkmark$ Publish Thesis/Dissertation as Monograph.

$\checkmark$ Publish Book Monograph.

$\checkmark$ Publish Edited Volume/ Book.

$\checkmark$ Publish Conference Proceedings

$\checkmark \quad$ Retain full copyright of your books.

Submit your manuscript at books. aijr.org 